Mathematical Modelling and Analysis

Volume 17 Number 1, February 2012, 90-99

http://dx.doi.org/10.3846/13926292.2012.645170

(c) Vilnius Gediminas Technical University, 2012
Publisher: Taylor\&Francis and VGTU

http://www.tandfonline.com/TMMA

Print ISSN: 1392-6292

Online ISSN: 1648-3510

\title{
Limit Theorems for Twists of $L$-Functions of Elliptic Curves. II
}

\author{
Virginija Garbaliauskienè $\dot{~}^{a}$ and Antanas Laurinčikas ${ }^{b}$ \\ ${ }^{a}$ Šiauliai University \\ P. Višinskio 19, LT-77156 Šiauliai, Lithuania \\ ${ }^{b}$ Vilnius University \\ Naugarduko 24, LT-03225 Vilnius, Lithuania \\ E-mail(corresp.): vgarbaliauskiene@gmail.com \\ E-mail: antanas.laurincikas@mif.vu.lt
}

Received June 2, 2011; revised November 5, 2011; published online February 1, 2012

\begin{abstract}
In the paper, a limit theorem for the argument of twisted with Dirichlet character $L$-functions of elliptic curves with an increasing modulus of the character is proved.
\end{abstract}

Keywords: Dirichlet character, elliptic curve, $L$-function of elliptic curve, probability measure, weak convergence.

AMS Subject Classification: 11M41; 44A15.

\section{Introduction}

In [3], we began to study limit theorems for twisted with Dirichlet character $L$-functions of elliptic curves with an increasing modulus of the character, and obtained a limit theorem of such a type for the modulus of these twists. Let $E$ be an elliptic curve over the field of rational numbers given by the Weierstrass equation

$$
y^{2}=x^{3}+a x+b, \quad a, b \in \mathbb{Z},
$$

with non-zero discriminant $\Delta=-16\left(4 a^{3}+27 b^{2}\right)$. For each prime $p$, denote by $E_{p}$ the reduction of the curve $E$ modulo $p$ which is a curve over the finite field $\mathbb{F}_{p}$, and define $\lambda(p)$ by

$$
\left|E\left(\mathbb{F}_{p}\right)\right|=p+1-\lambda(p),
$$

where $\left|E\left(\mathbb{F}_{p}\right)\right|$ is the number of points of $E_{p}$. The $L$-function $L_{E}(s), s=\sigma+i t$, of the elliptic curve $E$ is defined by the Euler product

$$
L_{E}(s)=\prod_{p \mid \Delta}\left(1-\frac{\lambda(p)}{p^{s}}\right)^{-1} \prod_{p \nmid \Delta}\left(1-\frac{\lambda(p)}{p^{s}}+\frac{1}{p^{2 s-1}}\right)^{-1} .
$$


Since, by the classical Hasse result,

$$
|\lambda(p)| \leqslant 2 \sqrt{p}
$$

for all primes, the product defining $L_{E}(s)$ converges uniformly on compact subset of the half-plane $\left\{s \in \mathbb{C}: \sigma>\frac{3}{2}\right\}$ and define there an analytic function without zeros. Moreover, in [1], the Taniyama-Shimura conjecture has been proved, therefore, the function $L_{E}(s)$ is analytically continued to an entire function, and satisfies the functional equation

$$
\left(\frac{\sqrt{N}}{2 \pi}\right)^{s} \Gamma(s) L_{E}(s)=w\left(\frac{\sqrt{N}}{2 \pi}\right)^{2-s} \Gamma(2-s) L_{E}(2-s),
$$

where, as usual, $\Gamma(s)$ denotes the Euler gamma-function, $N$ is the conductor of the curve $E$, and $w= \pm 1$.

The twist $L_{E}(s, \chi)$ with Dirichlet character $\chi$ for the function $L_{E}(s)$ is defined similarly. For $\sigma>\frac{3}{2}$, we have that

$$
L_{E}(s, \chi)=\prod_{p \mid \Delta}\left(1-\frac{\lambda(p) \chi(p)}{p^{s}}\right)^{-1} \prod_{p \nmid \Delta}\left(1-\frac{\lambda(p) \chi(p)}{p^{s}}+\frac{\chi^{2}(p)}{p^{2 s-1}}\right)^{-1},
$$

and function $L_{E}(s, \chi)$ is also analytically continued to an entire function.

Suppose that the modulus $q$ of the character $\chi$ is a prime number, and is not fixed. Denoting by $\chi_{0}$ the principal character modulo $q$, for $Q \geqslant 2$, define

$$
M_{Q}=\sum_{q \leqslant Q} \sum_{\substack{x=\chi(\bmod q) \\ \chi \neq \chi_{0}}} 1
$$

and put

$$
\mu_{Q}(\ldots)=M_{Q}^{-1} \sum_{q \leqslant Q} \sum_{\substack{x=\chi(\bmod q) \\ \chi \neq \chi_{0}}} 1
$$

where in place of dots we will write a condition satisfied by a pair $(q, \chi(\bmod q))$. Let $\mathcal{B}(S)$ stand for the class of Borel sets of the space $S$. Then in [3], the weak convergence of the frequency,

$$
\hat{P}_{Q}(A)=\mu_{Q}\left(\left|L_{E}(s, \chi)\right| \in A\right), \quad A \in \mathcal{B}(\mathbb{R}),
$$

as $Q \rightarrow \infty$, has been obtained. To state a limit theorem, we need some additional notation and definitions. For $p \nmid \Delta$, let $\alpha(p)$ and $\beta(p)$ be conjugate complex numbers such that $\alpha(p) \beta(p)=p$ and $\alpha(p)+\beta(p)=\lambda(p)$. Then (1.2), for $\sigma>\frac{3}{2}$, can be rewritten in the form

$$
L_{E}(s, \chi)=\prod_{p \mid \Delta}\left(1-\frac{\lambda(p) \chi(p)}{p^{s}}\right)^{-1} \prod_{p \nmid \Delta}\left(1-\frac{\alpha(p) \chi(p)}{p^{s}}\right)^{-1}\left(1-\frac{\beta(p) \chi(p)}{p^{s}}\right)^{-1} .
$$

As in [3], we use the notation $\eta=\eta(\tau)=i \tau / 2, \tau \in \mathbb{R}$, and, for primes $p$ and $k \in \mathbb{N}$,

$$
d_{\tau}\left(p^{k}\right)=\frac{\eta(\eta+1) \cdots(\eta+k-1)}{k !} .
$$


For $p \nmid \Delta$ and $k \in \mathbb{N}$, we set

$$
\begin{aligned}
& a_{\tau}\left(p^{k}\right)=\sum_{l=0}^{k} d_{\tau}\left(p^{l}\right) \alpha^{l}(p) d_{\tau}\left(p^{k-l}\right) \beta^{k-l}(p), \\
& b_{\tau}\left(p^{k}\right)=\sum_{l=0}^{k} d_{\tau}\left(p^{l}\right) \bar{\alpha}^{l}(p) d_{\tau}\left(p^{k-l}\right) \bar{\beta}^{k-l}(p),
\end{aligned}
$$

where $\bar{\alpha}(p)$ and $\bar{\beta}(p)$ denote the conjugates of $\alpha(p)$ and $\beta(p)$, respectively. For $p \mid \Delta$ and $k \in \mathbb{N}$, we define

$$
a_{\tau}\left(p^{k}\right)=b_{\tau}\left(p^{k}\right)=d_{\tau}\left(p^{k}\right) \lambda^{k}(p) .
$$

Let $a_{\tau}(m)$ and $b_{\tau}(m), m \in \mathbb{N}$, be multiplicative functions defined by (1.4)-(1.6), i.e.,

$$
a_{\tau}(m)=\prod_{p^{l} \| m} a_{\tau}\left(p^{l}\right), \quad b_{\tau}(m)=\prod_{p^{l} \| m} b_{\tau}\left(p^{l}\right),
$$

where $p^{l} \| m$ means that $p^{l} \mid m$ but $p^{l+1} \nmid m$.

On $(\mathbb{R}, \mathcal{B}(\mathbb{R}))$ define the probability measure $\hat{P}$ by the characteristic transforms [5],

$$
w_{k}(\tau)=\int_{\mathbb{R} \backslash\{0\}}|x|^{i \tau} \operatorname{sgn}^{k} \mathrm{~d} \hat{P}=\sum_{m=1}^{\infty} \frac{a_{\tau}(m) b_{\tau}(m)}{m^{2 \sigma}}, \quad \tau \in \mathbb{R}, k=0,1 .
$$

Theorem 1 [see [3]]. Suppose that $\sigma>\frac{3}{2}$. Then $\hat{P}_{Q}$ converges weakly to $\hat{P}$ as $Q \rightarrow \infty$.

The other results for $L$-functions with increasing modulus of the character are shortly discussed in [3].

The aim of this paper is to prove a limit theorem for the argument of the function $L_{E}(s, \chi)$. The estimate (1.1) and (1.3) show that $L_{E}(s, \chi) \neq 0$ for $\sigma>\frac{3}{2}$. Thus, for $\sigma>\frac{3}{2}, \arg L_{E}(s, \chi)$ is well defined. For $k \in \mathbb{Z}$, let $\theta=\theta(k)=\frac{k}{2}$, for primes $p$ and $l \in \mathbb{N}$,

$$
d_{k}\left(p^{l}\right)=\frac{\theta(\theta+1) \cdots(\theta+l-1)}{l !},
$$

and $d_{k}(1)=1$. Now similarly to (1.4) and (1.5), for $p \nmid \Delta$ and $l \in \mathbb{N}$, we define

$$
\begin{aligned}
& a_{k}\left(p^{l}\right)=\sum_{j=0}^{l} d_{k}\left(p^{j}\right) \alpha^{j}(p) d_{k}\left(p^{l-j}\right) \beta^{l-j}(p), \\
& b_{k}\left(p^{l}\right)=\sum_{j=0}^{l} d_{-k}\left(p^{j}\right) \bar{\alpha}^{j}(p) d_{-k}\left(p^{l-j}\right) \bar{\beta}^{l-j}(p) .
\end{aligned}
$$

If $p \mid \Delta$, then, for $l \in \mathbb{N}$, we set

$$
a_{k}\left(p^{l}\right)=d_{k}\left(p^{l}\right) \lambda^{l}(p), \quad b_{k}\left(p^{l}\right)=d_{-k}\left(p^{l}\right) \lambda^{l}(p) .
$$


Moreover, for $m \in \mathbb{N}$, we set

$$
a_{k}(m)=\prod_{p^{l} \| m} a_{k}\left(p^{l}\right), \quad b_{k}(m)=\prod_{p^{l} \| m} b_{k}\left(p^{l}\right) .
$$

Thus, $a_{k}(m)$ and $b_{k}(m)$ are multiplicative functions. Denote by $\gamma$ the unit circle on the complex plane. Furthermore, let $P$ be a probability measure on $(\gamma, \mathcal{B}(\gamma))$ defined by the Fourier transform

$$
g(k) \stackrel{\text { def }}{=} \int_{\gamma} x^{k} \mathrm{~d} P=\sum_{m=1}^{\infty} \frac{a_{k}(m) b_{k}(m)}{m^{2 \sigma}}, \quad k \in \mathbb{Z}, \sigma>\frac{3}{2} .
$$

The main result of this paper is the following statement.

Theorem 2. Suppose that $\sigma>\frac{3}{2}$. Then

$$
P_{Q}(A) \stackrel{\text { def }}{=} \mu_{Q}\left(\exp \left\{i \arg L_{E}(s, \chi)\right\} \in A\right), \quad A \in \mathcal{B}(\gamma),
$$

converges weakly to $P$ as $Q \rightarrow \infty$.

We recall that a distribution function $F(x)$ is said to be a distribution function $\bmod 1$ if

$$
F(x)= \begin{cases}1, & \text { if } x \geqslant 1 \\ 0, & \text { if } x<0\end{cases}
$$

Let $F_{n}(x), n \in \mathbb{N}$, and $F(x)$ be distribution functions $\bmod 1$. We say that $F_{n}(x)$, as $n \rightarrow \infty$, converges weakly $\bmod 1$ to $F(x)$, if at all continuity points $x_{1}, x_{2}, 0 \leqslant x_{1} \leqslant x_{2}<1$, of $F(x)$

$$
\lim _{n \rightarrow \infty}\left(F_{n}\left(x_{2}\right)-F_{n}\left(x_{1}\right)\right)=F\left(x_{2}\right)-F\left(x_{1}\right) .
$$

Denote by $L(s, \chi)$ the Dirichlet $L$-functions. Elliott in [2], for $\sigma>\frac{1}{2}$, obtained the weak convergence $\bmod 1$, as $Q \rightarrow \infty$, for

$$
\mu_{Q}\left(\frac{1}{2 \pi} \arg L(s, \chi) \leqslant x(\bmod 1)\right) .
$$

From Theorem 2, the following corollary follows.

Corollary 1. Suppose that $\sigma>\frac{3}{2}$. Then

$$
\mu_{Q}\left(\frac{1}{2 \pi} \arg L_{E}(s, \chi) \leqslant x(\bmod 1)\right)
$$

converges weakly mod 1 to the distribution function $\bmod 1$ defined by the Fourier transform $g(k)$ as $Q \rightarrow \infty$.

Differently from Dirichlet $L$-functions, we do not have any information on the convergence of the series defining the function $L_{E}(s, \chi), \chi \neq \chi_{0}$, in the region $\sigma>1$. Therefore, we can prove Theorem 2 only in the half-plane of absolute convergence of the mentioned series. Of course, we have a conjecture that the statement of Theorem 2 remains also true for $\sigma>1$, however, at the moment we can not prove this. 


\section{Fourier Transform}

Let $g_{Q}(k), k \in \mathbb{Z}$, denote the Fourier transform of $P_{Q}$ i.e., $g_{Q}(k)=\int_{\gamma} x^{k} \mathrm{~d} P$. Then the definition of $P_{Q}$ implies the equality

$$
g_{Q}(k)=\frac{1}{M_{Q}} \sum_{q \leqslant Q} \sum_{\substack{\chi=\chi(\bmod q) \\ \chi \neq \chi_{0}}} \mathrm{e}^{i k \arg L_{E}(s, \chi)} .
$$

For the proof of Theorem 2, we need the asymptotics of $g_{Q}(k)$ as $Q \rightarrow \infty$. In this section, we give an expression for $g_{Q}(k)$ convenient for the investigation of its asymptotics.

For any fixed $\delta>0$, denote by $R$ the region $\left\{s \in \mathbb{C}: \sigma \geqslant \frac{3}{2}+\delta\right\}$. For $s \in R$, we have that

$$
\begin{aligned}
\left(L_{E}(s, \chi)\right)^{\frac{1}{2}}\left(\overline{L_{E}(s, \chi)}\right)^{-\frac{1}{2}} & =\left(L_{E}(s, \chi)\right)^{\frac{1}{2}}\left(L_{E}(\bar{s}, \bar{\chi})\right)^{-\frac{1}{2}} \\
& =\left|L_{E}(s, \chi)\right|^{\frac{1}{2}} \mathrm{e}^{\frac{1}{2} i \arg L(s, \chi)}\left|L_{E}(s, \chi)\right|^{-\frac{1}{2}} \mathrm{e}^{-\frac{1}{2} i \arg L(s, \chi)} \\
& =\mathrm{e}^{i \arg L(s, \chi)} .
\end{aligned}
$$

Therefore, for $s \in R$ and $k \in \mathbb{Z} \backslash\{0\}$, formula (1.3) yields

$$
\begin{aligned}
& \mathrm{e}^{i k \arg L_{E}(s, \chi)}=\exp \left\{-\frac{k}{2} \sum_{p \mid \Delta}\left(\log \left(1-\frac{\lambda(p) \chi(p)}{p^{s}}\right)-\log \left(1-\frac{\lambda(p) \bar{\chi}(p)}{p^{\bar{s}}}\right)\right)\right. \\
& -\frac{k}{2} \sum_{p \nmid \Delta}\left(\log \left(1-\frac{\alpha(p) \chi(p)}{p^{s}}\right)+\log \left(1-\frac{\beta(p) \chi(p)}{p^{s}}\right)\right) \\
& \left.+\frac{k}{2} \sum_{p \nmid \Delta}\left(\log \left(1-\frac{\bar{\alpha}(p) \bar{\chi}(p)}{p^{\bar{s}}}\right)+\log \left(1-\frac{\bar{\beta}(p) \bar{\chi}(p)}{p^{\bar{s}}}\right)\right)\right\} \\
& =\prod_{p \mid \Delta} \exp \left\{-\theta \log \left(1-\frac{\lambda(p) \chi(p)}{p^{s}}\right)\right\} \\
& \times \prod_{p \nmid \Delta} \exp \left\{-\theta\left(\log \left(1-\frac{\alpha(p) \chi(p)}{p^{s}}\right)+\log \left(1-\frac{\beta(p) \chi(p)}{p^{s}}\right)\right)\right\} \\
& \times \prod_{p \mid \Delta} \exp \left\{\theta \log \left(1-\frac{\lambda(p) \bar{\chi}(p)}{p^{\bar{s}}}\right)\right\} \\
& \times \prod_{p \nmid \Delta} \exp \left\{\theta\left(\log \left(1-\frac{\bar{\alpha}(p) \bar{\chi}(p)}{p^{\bar{s}}}\right)+\log \left(1-\frac{\bar{\beta}(p) \bar{\chi}(p)}{p^{\bar{s}}}\right)\right)\right\} \\
& =\prod_{p \mid \Delta}\left(1-\frac{\lambda(p) \chi(p)}{p^{s}}\right)^{-\theta} \prod_{p \mid \Delta}\left(1-\frac{\lambda(p) \bar{\chi}(p)}{p^{\bar{s}}}\right)^{\theta} \\
& \times \prod_{p \nmid \Delta}\left(1-\frac{\alpha(p) \chi(p)}{p^{s}}\right)^{-\theta}\left(1-\frac{\beta(p) \chi(p)}{p^{s}}\right)^{-\theta}
\end{aligned}
$$




$$
\times \prod_{p \nmid \Delta}\left(1-\frac{\bar{\alpha}(p) \bar{\chi}(p)}{p^{\bar{s}}}\right)^{\theta}\left(1-\frac{\bar{\beta}(p) \bar{\chi}(p)}{p^{\bar{s}}}\right)^{\theta} .
$$

Here the multi-valued functions $\log (1-z)$ and $(1-z)^{ \pm \theta}$ in the region $|z|<1$ are defined by continuous variation along any path lying in this region from the values $\left.\log (1-z)\right|_{z=0}=0$ and $\left.(1-z)^{ \pm \theta}\right|_{z=0}=1$, respectively.

In the disc $|z|<1$, by the definition of $d_{k}\left(p^{l}\right)$ we have that

$$
(1-z)^{ \pm \theta}=\sum_{l=0}^{\infty} d_{\mp k}\left(p^{l}\right) z^{l}
$$

Therefore, (2.2) implies that, for $s \in R$,

$$
\begin{aligned}
\mathrm{e}^{i k \arg L_{E}(s, \chi)}= & \prod_{p \mid \Delta} \sum_{j=0}^{\infty} \frac{d_{k}\left(p^{j}\right) \lambda^{j}(p) \chi^{j}(p)}{p^{j s}} \prod_{p \nmid \Delta} \sum_{l=0}^{\infty} \frac{d_{k}\left(p^{l}\right) \alpha^{l}(p) \chi^{l}(p)}{p^{l s}} \\
& \times \sum_{v=0}^{\infty} \frac{d_{k}\left(p^{v}\right) \beta^{v}(p) \chi^{v}(p)}{p^{v s}} \prod_{p \mid \Delta} \sum_{j=0}^{\infty} \frac{d_{-k}\left(p^{j}\right) \lambda^{j}(p) \bar{\chi}^{j}(p)}{p^{j \bar{s}}} \\
& \times \prod_{p \nmid \Delta} \sum_{l=0}^{\infty} \frac{d_{-k}\left(p^{l}\right) \bar{\alpha}^{l}(p) \bar{\chi}^{l}(p)}{p^{l \bar{s}}} \sum_{v=0}^{\infty} \frac{d_{-k}\left(p^{v}\right) \bar{\beta}^{v}(p) \bar{\chi}^{v}(p)}{p^{v \bar{s}}} .
\end{aligned}
$$

Let $\hat{a}_{k}(m)$ and $\hat{b}_{k}(m)$ be multiplicative functions with respect to $m$ defined, for primes $p \nmid \Delta$ and $l \in \mathbb{N}$, by

$$
\begin{aligned}
& \hat{a}_{k}\left(p^{l}\right)=\sum_{j=0}^{l} d_{k}\left(p^{j}\right) \alpha^{j}(p) \chi\left(p^{j}\right) d_{k}\left(p^{l-j}\right) \beta^{l-j}(p) \chi\left(p^{l-j}\right), \\
& \hat{b}_{k}\left(p^{l}\right)=\sum_{j=0}^{k} d_{-k}\left(p^{j}\right) \bar{\alpha}^{j}(p) \bar{\chi}\left(p^{j}\right) d_{-k}\left(p^{l-j}\right) \bar{\beta}^{l-j}(p) \bar{\chi}\left(p^{l-j}\right),
\end{aligned}
$$

and, for primes $p \mid \Delta$ and $l \in \mathbb{N}$, by

$$
\hat{a}_{k}\left(p^{l}\right)=d_{k}\left(p^{l}\right) \lambda^{l}(p) \chi\left(p^{l}\right), \quad \hat{b}_{k}\left(p^{l}\right)=d_{-k}\left(p^{l}\right) \lambda^{l}(p) \bar{\chi}\left(p^{l}\right) .
$$

For $l \in \mathbb{N}$, we have that

$$
\begin{aligned}
\left|d_{ \pm k}\left(p^{l}\right)\right| & \leqslant \frac{|\theta|(|\theta|+1) \cdots(|\theta|+l-1)}{l !}=\theta \prod_{j=2}^{l}\left(1+\frac{|\theta|-1}{j}\right) \\
& \leqslant|\theta| \prod_{j=1}^{l}\left(1+\frac{|\theta|}{j}\right) \leqslant|\theta| \exp \left\{|\theta| \sum_{j=1}^{l} \frac{1}{j}\right\} \leqslant(l+1)^{c}
\end{aligned}
$$

where the constant $c$ depends on $k$, only. By the definition of $\alpha(p)$ and $\beta(p)$, we have that $|\alpha(p)|=|\beta(p)|=\sqrt{p}$. Therefore, for $p \nmid \Delta$ and $l \in \mathbb{N},(2.4)$ and (2.5) imply the bounds

$$
\left|\hat{a}_{k}\left(p^{l}\right)\right| \leqslant p^{\frac{l}{2}} \sum_{j=0}^{l}(j+1)^{c}(l-j+1)^{c} \leqslant p^{\frac{l}{2}}(l+1)^{2 c+1}
$$


and

$$
\left|\hat{b}_{k}\left(p^{l}\right)\right| \leqslant p^{\frac{l}{2}}(l+1)^{2 c+1} .
$$

It is known [4] that, for $p \mid \Delta$, the numbers $\lambda(p)$ are equal to 1 or 0 . Thus, by (2.6)-(2.7) we have that, for $p \mid \Delta$,

$$
\left|\hat{a}_{k}\left(p^{l}\right)\right| \leqslant(l+1)^{c}, \quad\left|\hat{b}_{k}\left(p^{l}\right)\right| \leqslant(l+1)^{c} .
$$

Now the multiplicativity of $\hat{a}_{k}(m)$ and $\hat{b}_{k}(m)$, and the estimates $(2.8)-(2.10)$ show that

$$
\begin{aligned}
& \hat{a}_{k}(m)=\prod_{p^{l} \| m}\left|\hat{a}_{k}\left(p^{l}\right)\right| \leqslant m^{\frac{1}{2}} \prod_{p^{l} \| m}(l+1)^{2 c+1}=m^{\frac{1}{2}} d^{2 c+1}(m), \\
& \left|\hat{b}_{k}(m)\right| \leqslant m^{\frac{1}{2}} d^{2 c+1}(m),
\end{aligned}
$$

where $d(m)$ is the divisor function. Since

$$
d(m)=\mathrm{O}_{\varepsilon}\left(m^{\varepsilon}\right)
$$

with every $\varepsilon>0$, the latter estimates imply, for every fixed $k \in \mathbb{Z} \backslash\{0\}$ and $s \in R$, the absolute convergence of the series

$$
\sum_{m=1}^{\infty} \frac{\hat{a}_{k}(m)}{m^{s}} \text { and } \sum_{m=1}^{\infty} \frac{\hat{b}_{k}(m)}{m^{s}} .
$$

Therefore, in view of (2.3), we conclude that, for every fixed $k \in \mathbb{Z} \backslash\{0\}$ and and $s \in R$,

$$
\begin{aligned}
\mathrm{e}^{i k \arg L_{E}(s, \chi)}= & \prod_{p \mid \Delta} \sum_{j=0}^{\infty} \frac{d_{k}\left(p^{j}\right) \lambda^{j}(p) \chi^{j}(p)}{p^{j s}} \prod_{p \nmid \Delta} \sum_{l=0}^{\infty} \frac{\hat{a}_{k}\left(p^{l}\right)}{p^{l s}} \\
& \times \prod_{p \mid \Delta} \sum_{j=0}^{\infty} \frac{d_{-k}\left(p^{j}\right) \lambda^{j}(p) \bar{\chi}^{j}(p)}{p^{j \bar{s}}} \prod_{p \nmid \Delta} \sum_{l=0}^{\infty} \frac{\hat{b}_{k}\left(p^{l}\right)}{p^{l \bar{s}}} \\
= & \prod_{p} \sum_{l=0}^{\infty} \frac{\hat{a}_{k}\left(p^{j}\right)}{p^{j s}} \prod_{p} \sum_{l=0}^{\infty} \frac{\hat{b}_{k}\left(p^{l}\right)}{p^{l \bar{s}}}=\sum_{m=1}^{\infty} \frac{\hat{a}_{k}(m)}{m^{s}} \sum_{n=1}^{\infty} \frac{\hat{b}_{k}(n)}{n^{\bar{s}}} .
\end{aligned}
$$

This and (2.1) give an expresion for the Fourier transform

$$
g_{Q}(k)=\frac{1}{M_{Q}} \sum_{q \leqslant Q} \sum_{\substack{x=\chi(\bmod q) \\ \chi \neq \chi_{0}}} \sum_{m=1}^{\infty} \frac{\hat{a}_{k}(m)}{m^{s}} \sum_{n=1}^{\infty} \frac{\hat{b}_{k}(n)}{n^{\bar{s}}} .
$$

\section{Proof of Theorem 2}

Having (2.14), we are in position to obtain the asymptotics for $g_{Q}(k)$ as $Q \rightarrow \infty$. First we modify the right-hand side of (2.14). Let $c_{1}=2 c+1$. Then, using (2.11)-(2.13), we find that, for $s \in R$, any fixed $k \in \mathbb{Z} \backslash\{0\}$ and $N \in \mathbb{N}$,

$$
\sum_{m>N} \frac{\hat{a}_{k}(m)}{m^{s}}=\mathrm{O}\left(\sum_{m>N} \frac{d^{c_{1}}(m)}{m^{1+\delta}}\right)=\mathrm{O}_{\varepsilon}\left(\sum_{m>N} \frac{1}{m^{1+\delta-\varepsilon}}\right)=\mathrm{O}_{\varepsilon}\left(N^{-\delta+\varepsilon}\right),
$$


and

$$
\sum_{m>N} \frac{\hat{b}_{k}(m)}{m^{\bar{s}}}=\mathrm{O}_{\varepsilon}\left(N^{-\delta+\varepsilon}\right) .
$$

Therefore, for any fixed $k \in \mathbb{Z} \backslash\{0\}$ and $s \in R,(2.14)$ can be rewritten as

$$
\begin{aligned}
& g_{Q}(k)=\frac{1}{M_{Q}} \sum_{q \leqslant Q} \sum_{\substack{\alpha=\chi(\bmod q) \\
\chi \neq \chi_{0}}}\left(\left(\sum_{m \leqslant N} \frac{\hat{a}_{k}(m)}{m^{s}}+\mathrm{O}_{\varepsilon}\left(N^{-\delta+\varepsilon}\right)\right)\right. \\
& \left.\times\left(\sum_{n \leqslant N} \frac{\hat{b}_{k}(n)}{n^{\bar{s}}}+\mathrm{O}_{\varepsilon}\left(N^{-\delta+\varepsilon}\right)\right)\right) \\
& =\frac{1}{M_{Q}} \sum_{q \leqslant Q} \sum_{\substack{\alpha=\chi(\bmod q) \\
\chi \neq \chi_{0}}}\left(\sum_{m \leqslant N} \frac{\hat{a}_{k}(m)}{m^{s}} \sum_{n \leqslant N} \frac{\hat{b}_{k}(n)}{n^{\bar{s}}}\right) \\
& +\mathrm{O}_{\varepsilon}\left(N^{-\delta+\varepsilon} \frac{1}{M_{Q}} \sum_{q \leqslant Q} \sum_{\substack{x=\chi(\bmod q) \\
\chi \neq \chi_{0}}}\left(\left|\sum_{m \leqslant N} \frac{\hat{a}_{k}(m)}{m^{s}}\right|+\left|\sum_{n \leqslant N} \frac{\hat{b}_{k}(n)}{n^{\bar{s}}}\right|\right)\right) \\
& +\mathrm{O}_{\varepsilon}\left(N^{-\delta+\varepsilon}\right) .
\end{aligned}
$$

Since, in view of (2.11)-(2.13), for any fixed $k \in \mathbb{Z} \backslash\{0\}$ and $s \in R$,

$$
\sum_{m \leqslant N} \frac{\hat{a}_{k}(m)}{m^{s}}=\mathrm{O}\left(\sum_{m=1}^{\infty} \frac{d^{c_{1}}(m)}{m^{1+\delta}}\right)=\mathrm{O}(1), \quad \sum_{n \leqslant N} \frac{\hat{b}_{k}(n)}{n^{\bar{s}}}=\mathrm{O}(1),
$$

we find that

$$
\frac{1}{M_{Q}} \sum_{q \leqslant Q}\left(\left|\sum_{\substack{\chi=\chi(\bmod q) \\ \chi \neq \chi_{0}}} \frac{\hat{a}_{k}(m)}{m^{s}}\right|+\left|\sum_{n \leqslant N} \frac{\hat{b}_{k}(n)}{n^{\bar{s}}}\right|\right)=\mathrm{O}(1) .
$$

Substituting this in (3.1), we obtain that, for any fixed $k \in \mathbb{Z} \backslash\{0\}$ and $s \in R$,

$$
g_{Q}(k)=\frac{1}{M_{Q}} \sum_{q \leqslant Q} \sum_{\substack{\chi=\chi(\bmod q) \\ \chi \neq \chi_{0}}}\left(\sum_{m \leqslant N} \frac{\hat{a}_{k}(m)}{m^{s}} \sum_{n \leqslant N} \frac{\hat{b}_{k}(n)}{n^{\bar{s}}}\right)+\mathrm{O}\left(N^{-\delta+\varepsilon}\right) .
$$

From the multiplicativity of the functions $\hat{a}_{k}(m)$ and $\hat{b}_{k}(m)$, and the complete multiplicativity of Dirichlet characters we deduce that

$$
\begin{aligned}
\hat{a}_{k}(m)= & \prod_{p^{l} \| m} \hat{a}_{k}\left(p^{l}\right)=\prod_{\substack{p^{l} \| m \\
p \nmid \Delta}}\left(\sum_{j=0}^{l} d_{k}\left(p^{j}\right) \alpha^{j}(p) \chi\left(p^{j}\right) d_{k}\left(p^{l-j}\right) \beta^{l-j}(p) \chi\left(p^{l-j}\right)\right) \\
& \times \prod_{\substack{p^{l} \| m \\
p \mid \Delta}} d_{k}\left(p^{l}\right) \lambda^{l}(p) \chi\left(p^{l}\right) \\
= & \left(\prod_{p^{l} \| m} \chi\left(p^{l}\right)\right) \prod_{\substack{p^{l} \| m \\
p \nmid \Delta}}\left(\sum_{j=0}^{l} d_{k}\left(p^{j}\right) \alpha^{j}(p) d_{k}\left(p^{l-j}\right) \beta^{l-j}(p)\right)
\end{aligned}
$$




$$
\times \prod_{\substack{p^{l} \| m \\ p \mid \Delta}} d_{k}\left(p^{l}\right) \lambda^{l}(p)=a_{k}(m) \chi(m)
$$

and similarly $\hat{b}_{k}(m)=b_{k}(m) \bar{\chi}(m)$, where the multiplicative functions $a_{k}(m)$ and $b_{k}(m)$ are defined in Section 1. Thus, (3.2) becomes

$$
g_{Q}(k)=\sum_{m \leqslant N} \frac{a_{k}(m)}{m^{s}} \sum_{n \leqslant N} \frac{b_{k}(n)}{n^{s}} \frac{1}{M_{Q}} \sum_{q \leqslant Q} \sum_{\substack{x=\chi(\bmod q) \\ \chi \neq \chi_{0}}} \chi(m) \bar{\chi}(n) .
$$

If $m=n$, then we have that

$$
\begin{aligned}
\sum_{q \leqslant Q} \sum_{\substack{\alpha=\chi(\bmod q) \\
\chi \neq \chi_{0}}} \chi(m) \bar{\chi}(n) & =\sum_{q \leqslant Q} \sum_{\substack{\chi=\chi(\bmod q) \\
\chi \neq \chi_{0}}}|\chi(m)|^{2}=M_{Q}-\sum_{\substack{q \mid m \\
q \leqslant N}}(q-2) \\
& =M_{Q}+\mathrm{O}\left(\sum_{q \leqslant N} q\right)=M_{Q}+\mathrm{O}\left(N^{2}\right) .
\end{aligned}
$$

Therefore, taking $N=\log Q$, and using the estimate [3]

$$
M_{Q}=\frac{Q^{2}}{2 \log Q}+\mathrm{O}\left(\frac{Q^{2}}{\log ^{2} Q}\right)
$$

as well as (2.11) and (2.12) type estimates for $a_{k}(m)$ and $b_{k}(m)$, we find that, for any fixed $k \in \mathbb{Z} \backslash\{0\}$ and $s \in R$,

$$
\begin{aligned}
& \sum_{\substack{m \leqslant N \\
m=n}} \sum_{n \leqslant N} \frac{a_{k}(m)}{m^{s}} \frac{b_{k}(n)}{n^{\bar{s}}} \frac{1}{M_{Q}} \sum_{q \leqslant Q} \sum_{\substack{\chi=\chi(\bmod q) \\
\chi \neq \chi_{0}}} \chi(m) \bar{\chi}(n) \\
& =\sum_{m \leqslant N} \frac{a_{k}(m) b_{k}(m)}{m^{2 \sigma}}(1+\mathrm{o}(1))=\sum_{m=1}^{\infty} \frac{a_{k}(m) b_{k}(m)}{m^{2 \sigma}}+\mathrm{o}(1)
\end{aligned}
$$

as $Q \rightarrow \infty$. It remains to consider the case $m \neq n$. For this, we will apply the relation

$$
\sum_{\chi=\chi(\bmod q)} \chi(m) \bar{\chi}(n)= \begin{cases}q-1 & \text { if } m \equiv n(\bmod q), \\ 0 & \text { if } m \neq \equiv(\bmod q),\end{cases}
$$

provided that $(m, q)=1$. So, for $m \neq n$ and $m, n \leqslant N$, we have that

$$
\begin{aligned}
& \sum_{q \leqslant Q} \sum_{\substack{\chi=\chi(\bmod q) \\
\chi \neq \chi_{0}}} \chi(m) \bar{\chi}(n)=\sum_{q \leqslant Q} \sum_{\chi=\chi(\bmod q)} \chi(m) \bar{\chi}(n)-\sum_{q \leqslant Q} \sum_{\chi=\chi_{0}(\bmod q)} \chi(m) \bar{\chi}(n) \\
& =\sum_{q \leqslant Q} \sum_{\substack{\chi=\chi(\bmod q) \\
q \mid(m-n)}} \chi(m) \bar{\chi}(n)+\sum_{\substack { q \leqslant Q \\
\begin{subarray}{c}{q=\chi(\bmod q) \\
q \nmid(m-n){ q \leqslant Q \\
\begin{subarray} { c } { q = \chi ( \operatorname { m o d } q ) \\
q \nmid ( m - n ) } }\end{subarray}} \chi(m) \bar{\chi}(n)+\mathrm{O}\left(\sum_{q \leqslant Q} 1\right) \\
& =\mathrm{O}\left(\sum_{q \leqslant N} q\right)+\mathrm{O}\left(\frac{Q}{\log Q}\right)=\mathrm{O}\left(\frac{Q}{\log Q}\right) .
\end{aligned}
$$


Therefore, we obtain that, for any fixed $k \in \mathbb{Z} \backslash\{0\}$ and $s \in R$,

$$
\begin{aligned}
& \sum_{\substack{m \leqslant N \\
m \neq n}} \sum_{\substack{n \leqslant N \\
m^{s} n^{\bar{s}}}} \frac{a_{k}(m) b_{k}(n)}{M_{Q}} \sum_{q \leqslant Q} \sum_{\substack{\chi=\chi(\bmod q) \\
\chi \neq \chi_{0}}} \chi(m) \bar{\chi}(n) \\
& =\mathrm{O}\left(\frac{1}{Q} \sum_{m \leqslant N} \frac{\left|a_{k}(m)\right|}{m^{\frac{3}{2}+\delta}} \sum_{m \leqslant N} \frac{\left|b_{k}(m)\right|}{m^{\frac{3}{2}+\delta}}\right) \\
& =\mathrm{O}\left(\frac{1}{Q}\left(\sum_{m \leqslant N} \frac{d^{c_{1}}(m)}{m^{1+\delta}}\right)^{2}\right)=\mathrm{o}(1)
\end{aligned}
$$

as $Q \rightarrow \infty$. Now this, (3.4) and (3.3) show that, for any fixed $k \in \mathbb{Z}$, uniformly in $s \in R$,

$$
g_{Q}(k)=\sum_{m=1}^{\infty} \frac{a_{k}(m) b_{k}(m)}{m^{2 \sigma}}+\mathrm{o}(1)
$$

as $Q \rightarrow \infty$. The last relation implies the weak convergence of $P_{Q}$ to the probability measure defined the Fourier transform

$$
\sum_{m=1}^{\infty} \frac{a_{k}(m) b_{k}(m)}{m^{2 \sigma}}
$$

as $Q \rightarrow \infty$. The same arguments also prove Corollary 1 .

\section{References}

[1] C. Breuil, B. Conrad, F. Diamond and R. Taylor. On the modularity of elliptic curves over $\mathbb{Q}$ : wild 3-adic exercises. J. Amer. Math. Soc., 14:843-939, 2001. http://dx.doi.org/10.1090/S0894-0347-01-00370-8.

[2] P.D.T.A. Elliott. On the distribution of $\arg L(s, \chi)$ in the half-plane $\sigma>\frac{1}{2}$. Acta Arith., 20:155-169, 1972.

[3] V. Garbaliauskienè, A. Laurinčikas and E. Stankus. Limit theorems for twist of $L$-functions of elliptic curves. Lith. Math. J., 50(2):187-197, 2010. http://dx.doi.org/10.1007/s10986-010-9079-z.

[4] H. Iwaniec and E. Kowalski. Analytic Number Theory. Amer. Math. Soc., Colleq. Publ., vol. 53, 2000.

[5] A. Laurinčikas. Remarks on the characteristic transforms of probability measures. Šiauliai Math. Semin., 2(10):43-52, 2007. 